\title{
Coronal activity cycles in 61 Cygni
}

\author{
A. Hempelmann ${ }^{1}$, J. Robrade ${ }^{1}$, J. H. M. M. Schmitt ${ }^{1}$, F. Favata ${ }^{2}$, S. L. Baliunas ${ }^{3}$, and J. C. Hall ${ }^{4}$ \\ 1 Universität Hamburg, Hamburger Sternwarte, Gojenbergsweg 112, 21029 Hamburg, Germany \\ e-mail: ahempelmann@hs .uni-hamburg.de \\ 2 Astrophysics Division - Research and Science Support Department of ESA, ESTEC, Postbus 299, 2200 AG Noordwijk, \\ The Netherlands \\ 3 Harvard-Smithsonian Center for Astrophysics, Cambridge, MA, USA \\ 4 Lowell Observatory, 1400 West Mars Hill Road, Flagstaff, AZ 86001, USA
}

Received 19 April 2006 / Accepted 25 July 2006

\section{ABSTRACT}

\begin{abstract}
Context. While the existence of stellar analogues of the 11 years solar activity cycle is proven for dozens of stars from optical observations of chromospheric activity, the observation of clearly cyclical coronal activity is still in its infancy.

Aims. In this paper, long-term X-ray monitoring of the binary 61 Cygni is used to investigate possible coronal activity cycles in moderately active stars.

Methods. We are monitoring both stellar components, a K5V (A) and a K7V (B) star, of 61 Cyg with XMM-Newton. The first four years of these observations are combined with ROSAT HRI observations of an earlier monitoring campaign. The X-ray light curves are compared with the long-term monitoring of chromospheric activity, as measured by the Mt.Wilson CaII $\mathrm{H}+\mathrm{K} S$-index.

Results. Besides the observation of variability on short time scales, long-term variations of the X-ray activity are clearly present. For $61 \mathrm{Cyg}$ A we find a coronal cycle which clearly reflects the well-known and distinct chromospheric activity cycle. The changes of coronal properties during the cycle resemble the solar behaviour. The coronal activity of $61 \mathrm{Cyg} \mathrm{B}$ also follows the chromospheric variability, although a pronounced sinusoidal chromospheric cycle of large amplitude is not noticeable. This is also reflected in the XMM-Newton observations with a rather complex long-term variability during that time.

Conclusions. $61 \mathrm{Cyg} \mathrm{A}$ is the first star where a persistent coronal activity cycle has been observed.
\end{abstract}

Key words. stars: activity - stars: chromospheres - stars: coronae - stars: late-type - X-rays: stars

\section{Introduction}

The question whether stellar activity cycles as observed in the light of CaII $\mathrm{H}+\mathrm{K}$ core emission (Baliunas et al. 1995) are also observable as X-ray cycles is not unambiguously answered up to date, mainly because of a lack of X-ray time series being long enough to cover a stellar cycle of a number of years. Additionally the differences in sensitivity and spectral coverage of the different X-ray observatories make it difficult to combine observations from various instruments. Studies of the Sun (Kreplin 1970), combined with characteristics of X-ray stellar observatories (cf., Peres et al. 2000, for ROSAT PSPC and ASCA SIS) revealed a pronounced solar coronal cycle of at least one order of magnitude variation between solar maximum and minimum (Peres et al. 2000). However, other sources of variability, such as flaring, emerging and decaying of active regions or rotational modulation can disturb the observability of a cycle, especially when only sparse snapshot observations are available.

A statistical study by Micela \& Marino (2003) using repetitive ROSAT/PSPC snapshot observations of solar-like main sequence stars and comparing their X-ray variations with the fulldisk solar X-ray variability transformed from $\mathrm{YOHKOH/SXT} \mathrm{to}$ the ROSAT/PSPC band showed active stars with $L_{\mathrm{X}}>10^{28} \mathrm{erg} / \mathrm{s}$ to be less variable than the Sun on long-time scales. Moderately active stars seem to be more variable on long time-scales and the authors concluded that at least a fraction of low-active stars could show solar-like coronal cycles. On the other hand it is known that the presence of well-defined chromospheric cycles is rather typical for low-active stars while strongly active stars tend to show an irregular long-term behaviour (Baliunas et al. 1995). Hempelmann et al. (1996) investigated the scatter around a relationship between $F_{\mathrm{X}}$ and the Rossby number for stars with known chromospheric cycles and correlated the residuals with the known phases of chromospheric cycles and found a weak correlation between chromospheric and X-ray activity.

While these indirect methods can only give hints to the existence of coronal cycles, the next step must be a long-term monitoring in X-rays over years and decades. The first star which was monitored with ROSAT over one magnetic cycle was EK Dra, a young and rapidly rotating solar analogue (Dorren et al. 1995; Güdel 2004). EK Dra shows no constant cycle in CaII H+K, however, the spot filling factor derived from light curve analysis of the rotating star varies periodically, indicating an activity cycle of 10.5 years (Järvinen et al. 2005). Its long term light curve shows tentative evidence for long X-ray variability in anti-phase to its visual light; the X-ray amplitude is about a factor of two.

Next, a long-term X-ray monitoring of the visual K5V/K7V binary 61 Cygni A+B with ROSAT HRI in the 1990s was correlated with Mt. Wilson CaII $\mathrm{H}+\mathrm{K}$ observations (Hempelmann et al. 2003, Paper I). The X-ray behaviour tracked the chromospheric variations in both stars, but the X-ray brightness variations were a factor of 2-3 smaller than on the Sun. However, the time basis of these observations was too short to cover a complete cycle. The conclusion from Paper I was that coronal cycles of 61 Cyg A and 61 Cyg B most probably do exist. 
Table 1. Observation log of $61 \mathrm{Cyg}$, duration of MOS1/PN.

\begin{tabular}{lccc}
\hline \hline Obs. ID & Obs. mode & Obs. time & Dur. (s) \\
\hline 0041740101 & LW/thick & 2002-05-14T11:22-13:38 & $550 / 5000$ \\
0041740301 & LW/thick & 2002-11-17T23:08-02:05 & $9270 / 7570$ \\
0041740901 & LW/thick & 2003-05-12T14:44-17:14 & $7670 / 6200$ \\
0041741001 & LW/thick & 2003-11-22T00:34-03:12 & $8170 / 6700$ \\
0041741101 & LW/thick & 2004-05-01T12:41-19:21 & $14250 / 12790$ \\
0043630101 & LW/thick & 2004-11-02T22:44-03:32 & $15970 / 13960$ \\
0043630401 & LW/thick & 2005-04-23T13:24-16:27 & $9670 / 7670$ \\
0202610401 & FF/thick & 2005-11-05T02:54-04:42 & $3540 / 5170$ \\
\hline
\end{tabular}

In order to study possible coronal activity cycles of moderate active stars in more detail, a long-term monitoring program of a small number of objects was initiated with XMM-Newton. Beside $61 \mathrm{Cyg}$, the monitored sample includes HD 81809 with first results published by Favata et al. (2004) and $\alpha$ Centauri with results presented by Robrade et al. (2005). HD 81809 and $\alpha$ Cen A exhibit order of magnitude variations of their X-ray brightness over several years, indicating the presence of coronal activity cycles. These stars are together with $61 \mathrm{Cyg}$ and EK Dra the only known examples with evidence for coronal activity cycles in solar-like stars so far. As in the case of $61 \mathrm{Cyg}$, the X-ray light curves showed a decreasing trend following the pronounced $\mathrm{CaII} \mathrm{H}+\mathrm{K}$ cycle. The amplitude of the X-ray luminosity variation in HD 81809 and $\alpha$ Cen A was larger by a factor of four than that of both $61 \mathrm{Cyg}$ A and $61 \mathrm{Cyg}$ B.

In this paper, we present the first four years of XMM-Newton observations of $61 \mathrm{Cyg} \mathrm{A}$ and B, combine them with the earlier ROSAT observations from Paper I and compare the coronal variability with the $\mathrm{CaII} \mathrm{H}+\mathrm{K}$ cycle.

\section{Observations}

\subsection{X-ray observations}

The target $61 \mathrm{Cyg}$ was observed twice a year with XMMNewton during the years 2002-2005. Using a nearly identical detector setup the exposure times range from 4-16 ks. We present EPIC (European Photon Imaging Camera) data from eight observations separated by roughly half a year each from our long-term monitoring program of moderately active stars. These measurements of the EPIC, and thus the MOS and PN detectors, allow us to investigate activity cycles with expected periods of several years and to check for short time variability during individual exposures. Exposure times vary between the instruments on-board XMM-Newton, a description of the observations is provided in Table 1, while a detailed description of the XMM-Newton instruments can be found in Ehle et al. (2003).

The data were reduced with the standard XMM-Newton Science Analysis System (SAS) software, version 6.0. Images, light curves and spectra were produced with standard SAS tools and standard selection criteria were applied for filtering the data, see Loiseau et al. (2004). Spectral analysis was carried out with XSPEC V11.3 (Arnaud 1996).

The analysis of possible coronal activity cycles is performed independently with MOS1 and PN data and consistent results are derived. The two components are well separated in all EPIC detectors and we use individual extraction regions with a $15^{\prime \prime}$ radius centered on each source to determine their X-ray properties. Figure 1 shows an X-ray image of $61 \mathrm{Cyg}$ as taken in Nov. 2002 with MOS1; the two source regions are indicated.

Both stars are moderately active stars and flaring is observed on both components. When investigating the cyclic behaviour

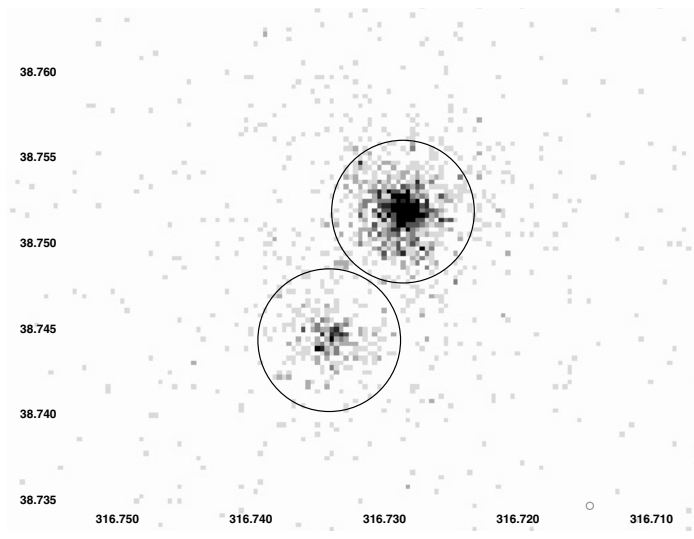

Fig. 1. Image of the binary 61 Cyg in Nov. 2002 obtained with MOS1. $61 \mathrm{Cyg}$ A (upper right) is near its coronal maximum in this exposure.
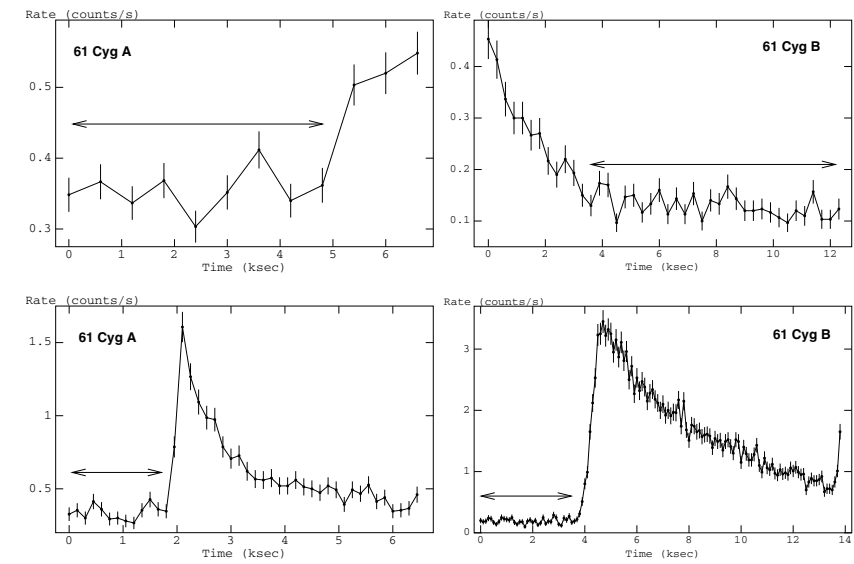

Fig. 2. Examples of light curves (PN) that are used for data screening; upper row: onset (left) and decay (right) of activity during an observation, bottom: flares. Quasi-quiescent time intervals are marked by arrows. $61 \mathrm{Cyg} \mathrm{B}$ exhibits more frequent and stronger variability than 61 Cyg A during our observations.

of $61 \mathrm{Cyg} \mathrm{A}$ and B, we considered only data from the quasiquiescent state. We excluded time periods of enhanced activity, i.e. where the flux from the respective component has increased abruptly from the quasi-quiescent state by about 50 or more percent, and stronger flares on the basis of the derived light curves for each individual exposure. Figure 2 shows some examples of light curves utilised for data selection, including the weakest activity considered and the strongest observed flaring. This reduces the effective observational time of some exposures, which is reflected in the errors of the individual observations. However, it is clear that events like stellar flares or strong activity must be rejected from a light curve of a stellar cycle.

The X-ray luminosities of the respective components during the individual exposures were derived from spectral analysis of EPIC data in the energy band between $0.2-2.0 \mathrm{keV}$, i.e. where sufficient signal in quasi-quiescence is present. To fit their X-ray spectra, we use a three-temperature model with solar abundances as given by Grevesse \& Sauval (1998) for each star and observation respectively. These models describe the spectra of both stars quite well. Other models were tested with lower or variable abundances and we find that the derived X-ray luminosities depend only marginally on the applied model. Deviations between different models are small compared to statistical errors and calibration uncertainties between MOS and PN. The applied model assumes the emission spectrum of a collisionally-ionized 
Table 2. X-ray luminosity $(0.2-2.0 \mathrm{keV})$ in $10^{26} \mathrm{erg} / \mathrm{s}$ determined from XMM-Newton data for $61 \mathrm{Cyg} \mathrm{A}$ and $\mathrm{B}$.

\begin{tabular}{lcc}
\hline \hline Obs. date & $L_{\mathrm{X}}(\mathrm{A})$ & $L_{\mathrm{X}}(\mathrm{B})$ \\
\hline $2002-05-14$ & $19.5 \pm 0.8$ & $7.6 \pm 0.5$ \\
$2002-11-17$ & $19.3 \pm 0.8$ & $6.9 \pm 0.4$ \\
$2003-05-12$ & $18.2 \pm 0.7$ & $7.2 \pm 0.5$ \\
$2003-11-22$ & $16.9 \pm 1.0$ & $6.2 \pm 0.6$ \\
$2004-05-01$ & $11.2 \pm 0.3$ & $6.5 \pm 0.4$ \\
$2004-11-02$ & $8.3 \pm 0.6$ & $6.0 \pm 0.4$ \\
$2005-04-23$ & $8.6 \pm 0.4$ & $6.5 \pm 0.4$ \\
$2005-11-05$ & $7.4 \pm 0.5$ & $8.0 \pm 0.5$ \\
\hline
\end{tabular}

optically-thin gas as calculated with the APEC code, see e.g. Smith et al. (2001). The background was taken from source free regions on the detectors and spectra were re-binned to a minimum value of 15 counts per spectral bin to satisfy the statistical demand of $\chi^{2}$ minimisation. From the respective best model we calculate the X-ray luminosities.

The spectra of both components are quite similar and the corresponding emission measure distributions (EMD) are dominated by relatively cool $(1-4 \mathrm{MK})$ plasma with variable contributions from a hotter component of about $8 \mathrm{MK}$. In Table 2 we give the calculated X-ray luminosities of $61 \mathrm{Cyg} \mathrm{A}$ and $\mathrm{B}$ as determined from MOS1 data (except May 2002 - MOS/PN data) for quasi-quiescent phases of the individual observations and adopting a distance of $3.48 \mathrm{pc}$. The given errors are Poissonian from the selected data of the respective observation.

The X-ray brightness of $61 \mathrm{Cyg} \mathrm{B}$ exhibits no clear trend throughout the XMM-Newton observations with irregular variations of less than $20 \%$ around a mean $L_{X}$ of $7 \times 10^{26} \mathrm{erg} / \mathrm{s}$.

In contrast, a clear decline in X-ray brightness is observed for $61 \mathrm{Cyg} \mathrm{A}$. We investigate possible accompanying spectral changes to test if a behaviour similar to the solar corona is present. On the Sun the average coronal temperatures drop but more importantly the emission measure decreases from solar maximum to minimum (see, e.g., Peres et al. 2000). Indeed, a similar behaviour is observed for the corona of $61 \mathrm{Cyg}$ A, supporting an activity cycle scenario as for the solar case. Three clear trends that accompany the X-ray darkening are observed for 61 Cyg A during the XMM-Newton observations. First, the hot plasma component around $8 \mathrm{MK}$ nearly vanishes towards activity minimum. Its contribution to the total emission measure is around $15 \%$ at activity maximum and decreases to about $3 \%$. Second, the average coronal temperature decreases from around $2.5 \mathrm{MK}$ to roughly $1.9 \mathrm{MK}$. This is of course related to the first point, but the average temperature also decreases within the cool plasma components that dominate the EMD. Third, the total emission measure decreases by a factor of two to three. This is much smaller than observed for the Sun, where the decrease in emission measure is about one order of magnitude. However, the variations in X-ray brightness for $61 \mathrm{Cyg} \mathrm{A}$ are also only of a factor of two to three. Comparing our values with those derived for the Sun in the ROSAT PSPC band by Peres et al. (2000), we find that the decrease in $L_{X}$ and emission measure over an activity cycle roughly scales by a similar factor for each star. This is due to the only moderate cooling of the coronae in each star by about $0.6 \mathrm{MK}$. Thus both stars exhibit very similar behaviour, despite their different spectral types of G 2 and K 5, the overall hotter corona of $61 \mathrm{Cyg} \mathrm{A}$ and the different cycle period. This suggests that the underlying mechanisms of activity cycles in moderately active cool stars are comparable.

A comparison is made with the earlier ROSAT HRI observations, with the ROSAT results taken from Paper I. The count

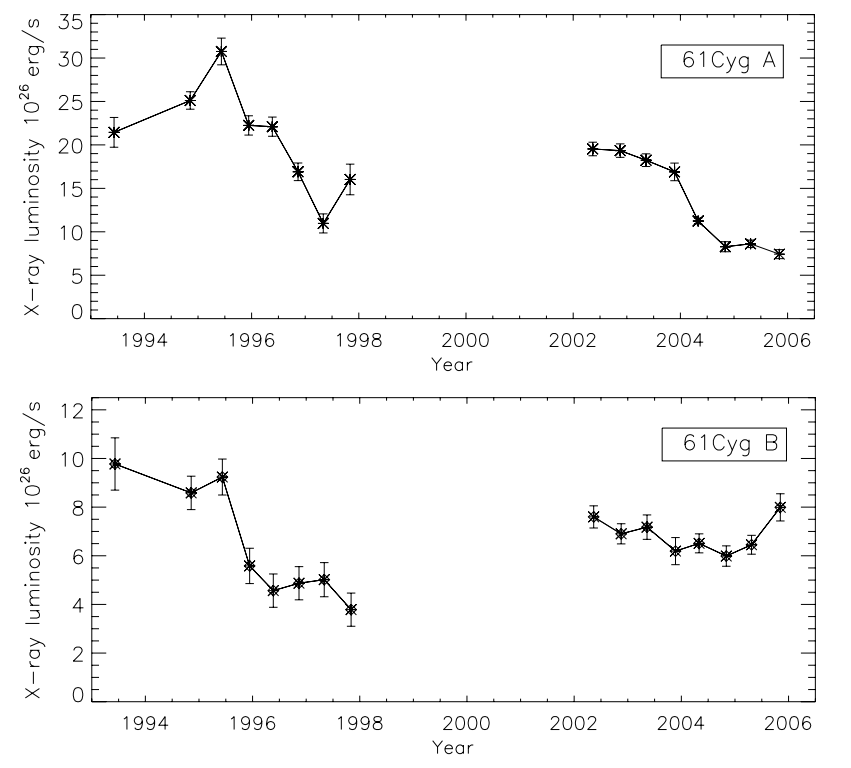

Fig. 3. X-ray light curves of $61 \mathrm{Cyg}$ A and B from the ROSAT and XMM-Newton observation program.

rate to flux conversion factor used in Paper I to derive the X-ray luminosities for the ROSAT data was cross-checked with the spectral models derived from the XMM-Newton observations. We find an agreement within $\sim 10 \%$, which is comparable to the statistical errors of the ROSAT data, but further uncertainties are present. We note that while we can rule out stronger flares for the ROSAT data, the short exposure times combined with the lower sensitivity of the HRI observations prevent an exclusion of periods affected by enhanced activity on a reliable basis for all data. Light curve variability and stronger flickering suggest especially the observations of 61 Cyg A during May 1995 and Oct. 1997 to be affected, see Fig. 3. We checked the impact of the presence of moderate activity by excluding only the stronger flares from the XMM-Newton data and find a slightly weaker correlation but no significant change in derived cycle period, amplitude and phase for these data. Further, calibration uncertainties may introduce an additional systematic error. From the investigation of other targets observed with ROSAT HRI and XMM-Newton we estimate this error to be up to $30 \%$. While this has no effect on periods and relative variations within each observation campaign, the absolute values may be affected as discussed in Sect. 3.

\subsection{Call $\mathrm{H}+\mathrm{K}$ observations}

$61 \mathrm{Cyg} \mathrm{A}+\mathrm{B}$ are stars included in Wilson's CaII H+K longterm monitoring program (Wilson 1968). Both stellar components were found to be cyclic with periods of 7.3 and 11.7 years for components A and B, respectively (Baliunas et al. 1995). Here we use the CaII $\mathrm{H}+\mathrm{K} S$ index (meaning and methods of determination are explained in Baliunas et al. 1995), in form of average $S$ index and average date, in 30-day bins.

The Mt. Wilson CaII $\mathrm{H}+\mathrm{K}$ project (MWO) was closed in 2002. Before its closing a follow-up program was started at Lowell Observatory in 1992. The Lowell project monitors 143 stars, including the Sun and many of the MWO stars; stellar observations are made using a 0.9 -m telescope and a $R \approx 12000$ spectrograph, including the HK region from 3860-4010 $\AA$ and focusing primarily on solar analogues $(\approx 0.55 \leq B-V \leq 0.72)$ of $V<7.5$. 


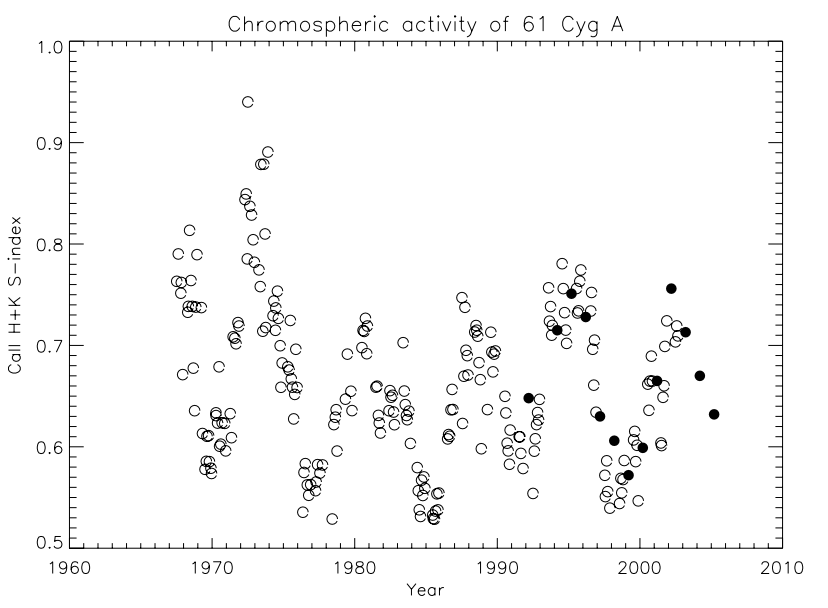

Fig. 4. 61 Cyg A: Mt. Wilson CaII $\mathrm{H}+\mathrm{K}$ emission core measures (open circles) in combination with observations from Lowell Observatory (filled circles). The observations are transformed to the Mt. Wilson $S$ index.

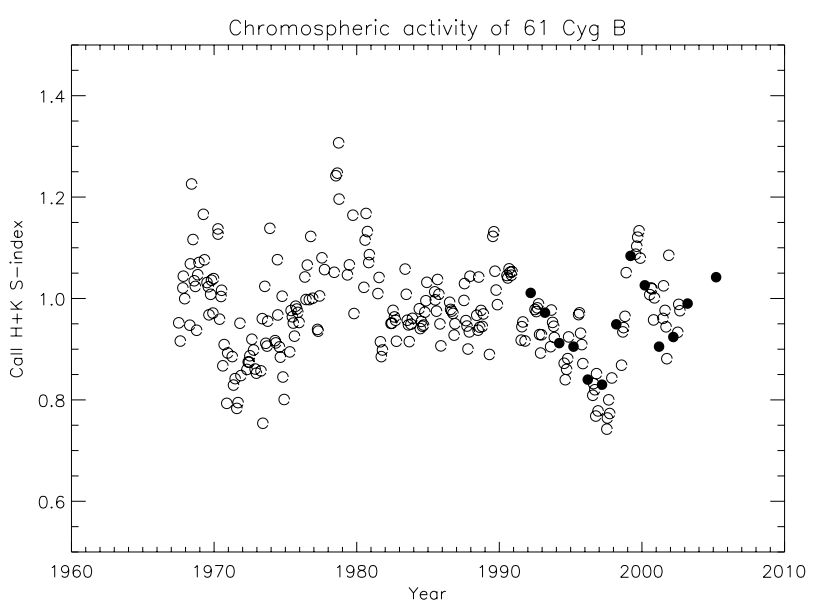

Fig. 5. 61 Cyg B: for more details see Fig. 4.

The total time series of emission core CaII $\mathrm{H}+\mathrm{K} S$ index observations (in the following abbreviated with CaHK) of 61 Cyg A is depicted in Fig. 4. Mt. Wilson observations are assigned with open circles, Lowell observations with full circles. There is no systematic shift between the two data sets, so one can expect that the Lowell observations alone (after 2002) correctly describe the chromospheric activity of $61 \mathrm{Cyg}$ A. However, the data sampling of the Lowell observations is sparse in comparison with the Mt. Wilson data so that the few observations after 2002 might reflect variability on shorter time-scales rather than the well-known chromospheric cycle of 7.3 years (Baliunas et al. 1995). On the other hand, the cycle of this star is very pronounced and dominates over short-term fluctuations. The cycle period and amplitude are not strongly varying and suggest there should be a decreasing trend of activity after 2002 which is visible in the Lowell data. Therefore we regard this trend as reliable.

The picture of $61 \mathrm{Cyg} \mathrm{B}$ is not so clear (see Fig. 5). On the one hand, there is no excellent cycle visible, on the other hand, a Fourier spectrum of this time-series exhibits a clearly dominant period of $11.2 \mathrm{yr}$ (which is half a year shorter than the period of $11.7 \mathrm{yr}$ found by Baliunas et al. (1995); their estimate was based on a shorter time-series). Furthermore, the Lowell observations have a gap in 2004. The 2004 gap in the observations exists because the telescope was more or less completely rebuilt at that time, including installation of new optics. Unfortunately,

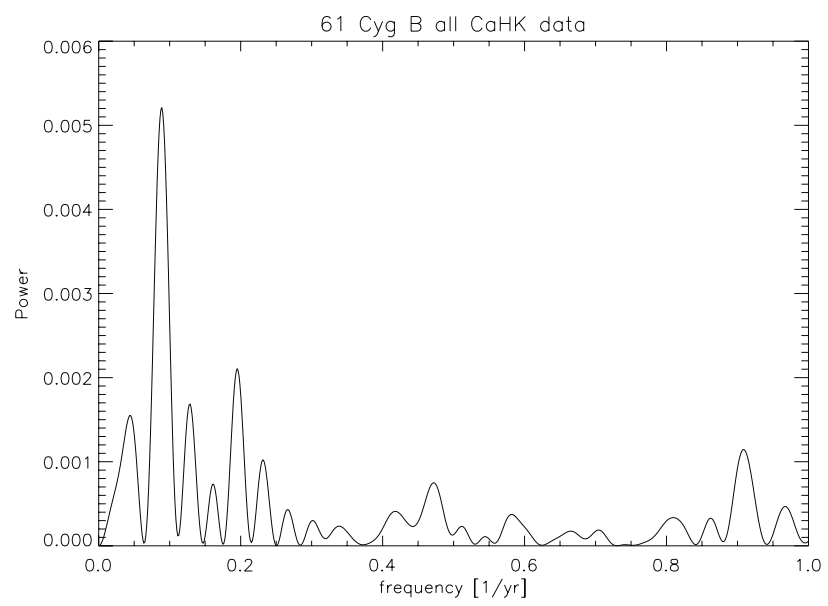

Fig. 6. 61 Cyg B: Fourier spectrum of the $\mathrm{Ca} \mathrm{H}+\mathrm{K}$ time-series depicted in Fig. 5.

the lengthy downtime coincided with the 61 Cyg observing season. Thus it is not quite clear whether the activity shows really a trend of increasing activity suggested by the Lowell observation in 2005 or is of a chaotically fluctuating behaviour without any trend as seen in the eighties. The eleven years cycle will mainly result from the four observed maxima followed by a descent, whereas the two clear minima visible in the early seventies and late nineties followed by a strong increase indicate a much longer period of about 25 years which is not prominent in Fig. 6. However, similarities in the structures after these two minima suggest a plateau after 2003 as seen in the eighties rather than a rise. Nevertheless we have to conclude that we are not able to deduce a unique picture of chromospheric activity behaviour for the times of XMM-Newton observations.

\section{Results: activity cycles}

\subsection{Cygni $A$}

The time span between the ROSAT and the XMM-Newton observations of about 4 years corresponds to roughly half a chromospheric activity cycle of 61 Cyg A. The ROSAT HRI observations were started in 1993, two years before the CaHK activity had reached its maximum in 1995, and had been finished in late 1997, roughly two and a half years after CaHK activity maximum. The chromospheric cycle of this star appeared slightly asymmetric in those years. The minimum was reached already during 1998 shortly after the end of the ROSAT HRI observations (see Fig. 8). XMM-Newton measurements began at the maximum of the following activity cycle in 2002. The last measurement reported in this paper was carried out in late 2005, i.e. the XMM-Newton campaign covered less than half an activity cycle and therefore should sample the descending branch of activity. Unfortunately, the gap between the two observational campaigns covers exactly the ascending branch of the activity cycle and there are no XMM-Newton observations from phases of clearly increasing activity. On the other hand, this special (by chance) data sampling allows a direct comparison of the amplitudes and mean activity levels because systematic $L_{X}$ shifts between ROSAT and XMM-Newton observations could not be explained by different phase coverages.

In both series, the X-ray amplitude between minimum and maximum activity is a factor of three (Fig. 3). We note, however, that the cycle amplitude is much smaller compared to those of the Sun and HD 81809 (Favata et al. 2004), while the 

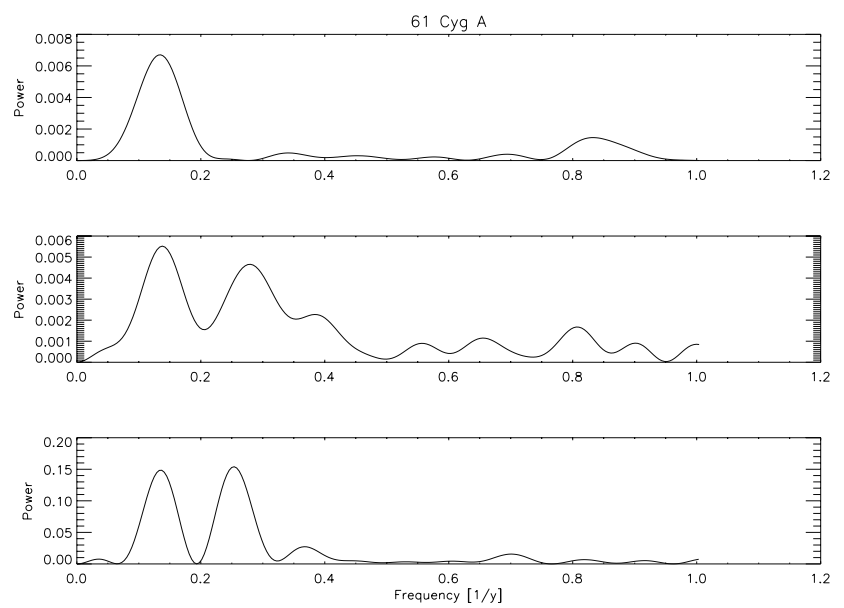

Fig. 7. Fourier spectra of 61 Cyg A. Top: CaHK between 1993 and 2005. Middle: $\mathrm{CaHK}$ data with a similar sampling as for the X-ray data. Bottom: X-ray data of ROSAT and XMM-Newton.

chromospheric activity of $61 \mathrm{Cyg}$ A is much higher than for the Sun and HD 81809. The ROSAT HRI measurements indicate a $30 \%$ higher X-ray luminosity during the maximum in 1995 , in comparison with the maximum in 2002 . This could be a real variation in the cycle amplitude, as might be suggested by the CaHK data (see Fig. 4). Another possibility is cross-calibration uncertainty between the two X-ray instruments.

In order to overcome the problem of a possible calibration uncertainty and to be able to compare the different measures of activity and the different scales of variability in a simple manner, we have transformed both the X-ray luminosities of ROSAT and XMM-Newton as well as the CaHK data on values relative to their respective means (Fig. 8, open circles and full dots). Then, Fourier analysis was carried out and the coronal activity was compared with the chromospheric cycle in their phase relationship. The result of the Fourier analysis is shown in Fig. 7. The spectrum of the X-ray time-series (bottom of Fig. 7) is characterised by two enhanced peaks of almost equal power at frequencies 0.135 and 0.254 cycles per year, corresponding to periods of 7.4 and 3.9 years. At top part of Fig. 7, the spectrum of that part of the CaHK time series which span only the interval between the beginning of the ROSAT observations and the end of the XMM-Newton observations is shown. This spectrum shows only a single enhanced peak of frequency 0.135 cycles/year which is exactly the same frequency as found for one of the two possible coronal cycles.

The appearance of a second possible cycle in the coronal Fourier spectrum could have two reasons: i) it is the first harmonic; ii) it results from data sampling. The first explanation is not so likely because the frequency deviates somewhat from the half. To check the second explanation, we extracted a $\mathrm{CaHK}$ time series which consists only of data points measured closest in time to the X-ray observations. The resulting Fourier spectrum is shown in the middle of Fig. 7. The double-peak structure as seen for the X-ray data is now conspicuous also in the CaHK data when sampled in a similar manner as for the X-ray data. We suggest that the double solution for the X-ray data likely results from its sampling. A unique solution requires observations during the phase of increasing activity.

We have to note that from our somewhat ill-sampled X-ray observations alone a unique cycle period is not derivable but an unambiguous solution can be expected from a complete coverage of a full cycle. However, we know the period of the activity

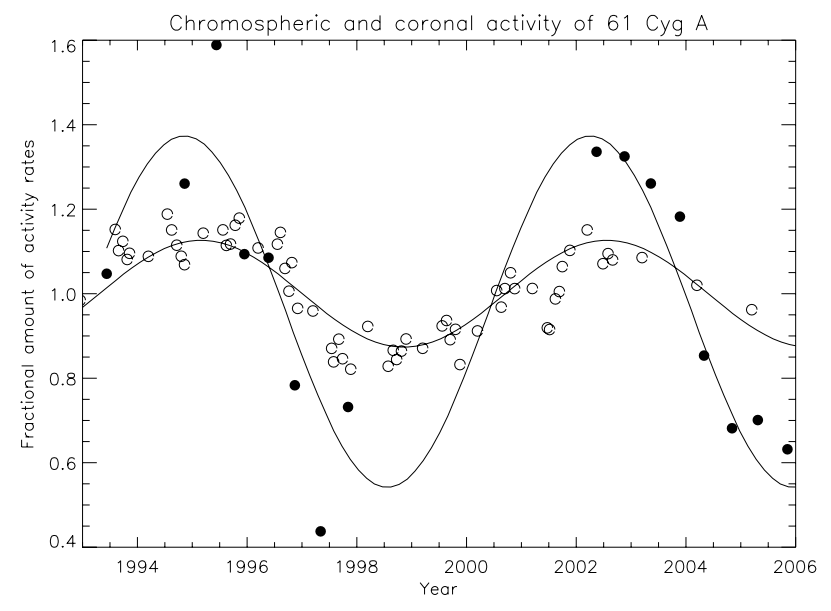

Fig. 8. 61 Cyg A: comparison between chromospheric (open circles) and coronal activity (full dots). The continuous curves are least squares sine fits.

cycle from the chromospheric observations. Hence, we can also limit our X-ray solution to the 7.4 years cycle.

On the basis of this solution we have fitted the X-ray data as well as the CaHK data of Fig. 8 with a sine and estimated the amplitudes and times of maxima. The fits are shown in Fig. 8 as solid lines. The times of X-ray fit maxima (1994.9 and 2002.3) are nearly perfectly in congruence with the fit maxima of chromospheric activity: 1995.1 and 2002.5. Note, however, that the chromospheric cycle is indeed slightly asymmetric resulting in a (slight) error when fitting with a pure sine. So the pure sine fit shifts its minimum clearly a bit to the right from the true data minimum which could explain the small difference of $0.2 \mathrm{yr}$ between the coronal and the chromospheric fit. Nevertheless, there is no doubt that both cycles are close together in phase.

\subsection{Cygni B}

The chromospheric cycle of $61 \mathrm{Cyg} B$ is less smooth than that of $61 \mathrm{Cyg} \mathrm{A}$ and characterised by more irregular variations (see Fig. 5). It is also not clear from the CaHK data whether there is a trend of increasing activity after 2002 as suggested mainly from the data point in 2005. It cannot be excluded that the chromospheric activity actually remained flat (but fluctuating) during those times. A similar behaviour is found in the X-ray data which also indicate a kind of plateau and are slightly increasing towards the end of 2005.

We note that the $S$-index of $61 \mathrm{Cyg}$ B is higher than that of $61 \mathrm{Cyg}$ A and most other stars observed in the whole Mt. Wilson program. Further, its chromospheric activity cycle is neither smooth nor symmetric over the last decades. This is reflected in the X-ray behaviour of $61 \mathrm{Cyg} \mathrm{B}$, which is characterised by frequent short-term activity and flaring over the time period covered by the XMM-Newton observations.

For further investigations all the data were transformed to values relative to their respective means as was done for component A. These transformed time-series are depicted in Fig. 10. There the CaHK data are plotted as circles, the X-ray data as full dots, and the continuous curves are second-order Fourier fits (Eq. (1)). The corresponding Fig. 9 shows the result of a Fourier analysis which was done in the same manner as for component A. The maximum peak for the CaHK data of Fig. 9 (top) appears at frequency 0.11 cycles/yr corresponding a period of 9.1 years. This lies remarkably far away from the $11.2 \mathrm{yr}$ period 

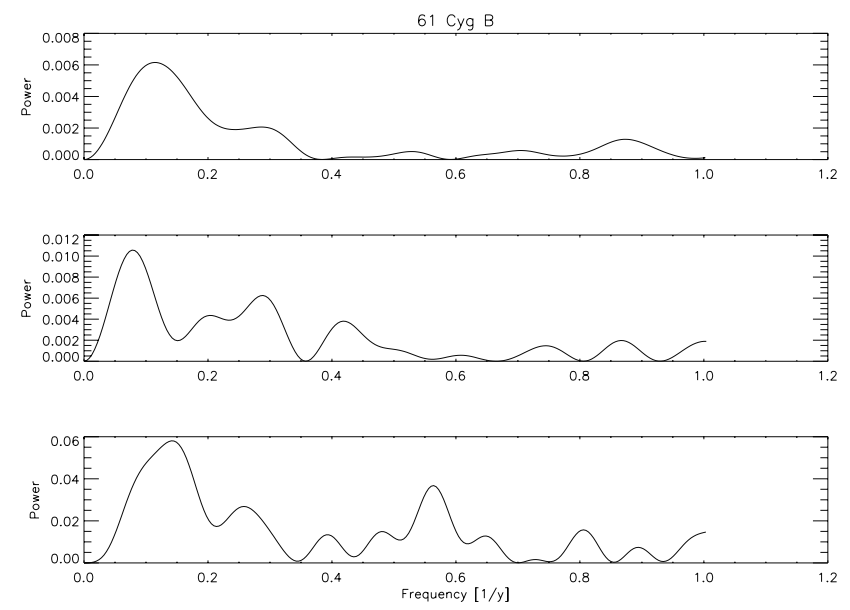

Fig. 9. Fourier spectra of $61 \mathrm{Cyg}$ B. Top: CaHK between 1993 and 2005. Middle: CaHK data with a similar sampling as for the X-ray data. Bottom: X-ray data of ROSAT and XMM-Newton.

of the whole time-series of Fig. 5. However, this peak is very broad and may partly reflect the above mentioned fluctuating behaviour.

A Fourier analysis of the X-ray data (bottom of Fig. 9) shows a similar broad peak as for the the CaHK data but this peak appears asymmetric and more like a blend. When the CaHK data are sampled according to the X-ray data sampling (middle of Fig. 9), this Fourier spectrum has neither strong similarity with the X-ray spectrum nor with the CaHK spectrum of the nonreduced (top of Fig. 9) data set. The sampling does not seem to be the problem as it is for component A.

The CaHK time-series shows clear and strong deviation from a pure sine (Fig. 5). Thus we fitted both the $\mathrm{CaHK}$ and the X-ray time-series with second order trigonometrical polynomials:

$$
\begin{aligned}
y(t)= & \cos (2 \pi / P \cdot t)+\sin (2 \pi / P \cdot t) \\
& +\cos (4 \pi / P \cdot t)+\sin (4 \pi / P \cdot t)
\end{aligned}
$$

where $P$ is the cycle period of 11.2 years, $\mathrm{t}$ is the time, and $y$ is the fractional amount of activity rates either in X-rays or in CaHK. The result is shown in Fig. 10 as solid lines. It is obvious that both curves are similar in shape, but different in amplitudes and also somewhat different in phase. While the CaHK curve varies between minimum and maximum by about $20-30 \%$, the coronal activity varies roughly by a factor of two. This is smaller in both activity indicators than for component A. (Note that component $\mathrm{B}$ is the chromospherically more active star.) The X-ray fit curve is one year later in phase as for the CaHK fit curve (as it is visible from Fig. 10). This might reflect a real phase shift but we do not claim this as a significant result because of the uncertainties resulting from the large data gap and the complex character of variability of this star.

\section{Conclusions}

The XMM-Newton observations of $61 \mathrm{Cyg}$, in combination with earlier ROSAT HRI observations, confirm the existence of a coronal activity cycle for $61 \mathrm{Cyg} \mathrm{A}$. This is to our knowledge the first persistent stellar coronal activity cycle yet detected. The $\mathrm{X}$-ray light curve is well correlated and in phase with the ROSAT observation from the 1990s as well as the CaHK measurements. The corona of $61 \mathrm{Cyg}$ A is a little hotter than that of the Sun; however, its changes during the activity cycle are comparable to the Sun's, i.e., a decrease of the total emission measure is

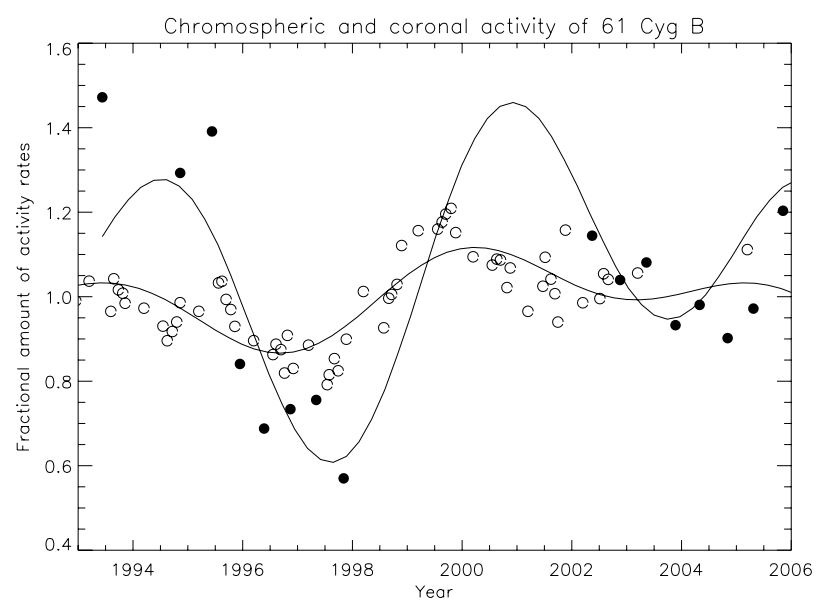

Fig. 10. 61 Cyg B: comparison between chromospheric (open circles) and coronal activity (full dots). The continuous curves are least squares second-order Fourier fits basing of the long-term chromospheric cycle of $11.2 \mathrm{yr}$.

accompanied by a moderate decline of the average coronal temperature. Furthermore, the cycle amplitude is a factor of about three smaller than that of the Sun or other stars in our program (HD 81809, $\alpha$ Cen A), while the chromospheric $S$-index is much higher. With $\left\langle\log L_{X} / L_{\text {bol }}\right\rangle=-5.5$, the level of coronal activity is also higher than for the Sun for which $\log L_{\mathrm{X}} / L_{\mathrm{bol}}$ transformed to the ROSAT passband was determined to be variable between -6.9 and -5.5 over a solar cycle (Judge et al. 2003). Component $\mathrm{B}$ is also more active $\left(\left\langle\log L_{\mathrm{X}} / L_{\mathrm{bol}}\right\rangle=-5.8\right)$ than the Sun but shows a lower level of coronal activity than component A while its chromospheric activity (Noyes et al. 2003) is $25 \%$ higher than that of $\mathrm{A}$.

Of course, more detailed conclusions on the general properties of the relation between chromospheric and coronal activity cycles cannot be claimed from a sample of only a few stars. However, the probability of finding a coronal cycle will surely depend on the characteristics of long-term activity and the CaHK measurements have been proven to be a good indicator for coronal activity cycles. Stars with moderate chromospheric activity levels and distinct $\mathrm{CaHK}$ cycles like $61 \mathrm{Cyg}$ A are naturally better candidates for detectability of coronal cycles than more active stars with a rather complex long-term activity behaviour like 61 Cyg B. However, the coronal behaviour is also quite well in phase with chromospheric variability for $61 \mathrm{Cyg}$ B although this star shows a more complex pattern of lang-term activity with a rather flat curve during the XMM observations.

A further aspect is, of course, data sampling. First, occasional observations (as is frequently the case for space-based observations) can fall by chance in phases of low variability. Second, large gaps in a time-series (which is also rather the standard for X-ray observations) can contaminate a result or make a solution ambiguous. A good example for such a case is our finding for $61 \mathrm{Cyg}$ A. We observed two series of X-ray observations when the chromospheric activity of $61 \mathrm{Cyg}$ A coincidentally decreased from a maximum to the next minimum. In both cases the amplitude of this change is much higher in X-rays than in $\mathrm{CaHK}$, indicating that the coronal cycle of this star is still more pronounced than the already distinct chromospheric cycle. However, because of a gap between the two time-series of ROSAT and XMM-Newton, there are two solutions possible concerning the period of the coronal cycle: the true period (well known from the CaHK time-series) and a second period about 
half as long. The latter almost certainly results from the (natural) gap between finishing the ROSAT operations and starting the XMM-Newton monitoring program. Our simple conclusion from this experience is that the XMM-Newton monitoring program should be continued into the following phase of expected increasing activity of $61 \mathrm{Cyg} A$ at least up to the next maximum to obtain an unambiguous solution for the X-ray cycle period.

Acknowledgements. This work is based on observations obtained with XMMNewton, an ESA science mission with instruments and contributions directly funded by ESA Member States and the USA (NASA).

J.R. acknowledges support from DLR under 50OR0105. J.C.H. acknowledges support from the National Science Foundation under grant ATM-0447159.

\section{References}

Arnaud, K. A. 1996, ASP Conf. Ser., 101, 17

Baliunas, S. L., Donahue, R. A., Soon, W. H., et al. 1995, ApJ, 438, 269

Dorren, J. D., Güdel, M., \& Guinan, E. F. 1995, ApJ, 448, 431
Ehle, M., Breitfellner, M., Gonzales Riestra, M., et al. 2003, XMM-Newton User's Handbook

Favata, F., Micela, G., Baliunas, S. L., et al. 2004, A\&A, 418, L13

Grevesse, N., \& Sauval, A. J. 1998, SSR, 95, 161

Güdel, M. 2004, A\&ARv, 12, 71

Hempelmann, A., Schmitt, J. H. M. M., \& Stępień, K. 1996, A\&A, 305, 284

Hempelmann, A., Schmitt, J. H. M. M., Baliunas, S. L., \& Donahue, R. A. 2003, A\&A, 406, L39 (Paper I)

Järvinen, S. P., Berdyugina, S. V., \& Strassmeier, K. G. 2005, A\&A, 440, 735

Judge, P. G., Solomon, S. C., \& Ayres, T. R. 2003, ApJ, 593, 534

Kreplin, R. W. 1970, Annales de Geophysique, 26, 567

Loiseau, N., Ehle, M., Pollock, A. M. T., et al. 2004, User's Guide to XMMNewton Science Analysis System Issue 3.1

Micela, G., \& Marino, A. 2003, A\&A, 404, 637

Noyes, R. W., Hartmann, L. W., Baliunas, S. L., Duncan, D. K., \& Vaughan, A. H. 1984, ApJ, 279, 763

Peres, G., Orlando, S., Reale, F., Rosner, R., \& Hudson, H. 2000, ApJ, 528, 537

Robrade, J., Schmitt, J. H. M. M., \& Favata, F. 2005, A\&A, 442, 315

Smith, R. K., Brickhouse, N. S., Liedahl, D. A., \& Raymond, J. C. 2001, ASP Conf. Ser., 247, 161

Wilson, O. C. 1968, ApJ, 153, 221 